\title{
Eradication of small intestinal bacterial overgrowth in irritable bowel syndrome using herbal therapy: A case report
}

\author{
CIARA P. WRIGHT ${ }^{1}$, MELISSA T. DOOLEY ${ }^{2}$ and HEATHER LEESON ${ }^{1}$ \\ ${ }^{1}$ Glenville Nutrition, Dublin D06 T265; ${ }^{2}$ GastroLife, Dublin 18 D18 E7P4, Ireland
}

Received September 15, 2021; Accepted December 2, 2021

DOI: $10.3892 / \mathrm{ijfn} .2021 .23$

\begin{abstract}
Small intestinal bacterial overgrowth (SIBO) is a common pathology in irritable bowel syndrome (IBS). Symptoms include bloating, excessive belching, flatulence, epigastric and abdominal pain, nausea, early satiety, fatigue and altered bowel habits. Given the high incidence of IBS globally, SIBO needs to be investigated in IBS using hydrogen and methane breath testing. Adherence to the North American Consensus 2017 or the updated guidelines from the Association of Gastrointestinal Physiology (AGIP) committee of the British Society of Gastroenterology 2019 is necessary to ensure that testing and diagnosis are standardised. The treatment of SIBO with antibiotics, most commonly rifaximin, may restore gastrointestinal function and reduce the symptoms of IBS. A number of herbal and nutraceutical supplements are known to exert antimicrobial effects. There is increasing awareness amongst the public regarding these herbal and nutraceutical supplements, and there is an increased demand for their use. The present study describes the case of a patient with severe IBS symptoms, scoring in the extreme category on a quality of life questionnaire validated for IBS and functional digestive disorders. The patient tested positive for SIBO, which was then eradicated using a herbal formulation containing garlic, berberine, oregano, grapefruit seed extract and caprylic acid, while following a diet low in fermentable oligo-, di-, mono-saccharides and polyols (FODMAP). The patient's quality of life assessment markedly improved following treatment and the remaining symptoms improved at the 3-month follow-up performed by verbal confirmation.
\end{abstract}

\section{Introduction}

Irritable bowel syndrome (IBS) is a functional and often debilitating disorder of the gastrointestinal tract. It can be characterised by IBS with diarrhoea (IBS-D), IBS with

Correspondence to: Dr Ciara P. Wright, Glenville Nutrition, 10 Orwell Road, Rathgar, Dublin D06 T265, Ireland

E-mail: ciara.wright@glenvillenutrition.ie

Key words: small intestinal bacterial overgrowth, irritable bowel syndrome, hydrogen and methane breath test, herbal antimicrobial constipation (IBS-C) or mixed constipation and diarrhoea (IBS-M) (1). A diagnosis of IBS is made based on the patient's symptoms of abdominal pain and altered bowel habits, using the 2016 Rome criteria guidelines, ROME IV (2). IBS affects $\sim 11 \%$ of the population globally (1).

The underlying causes of IBS are likely multifactorial, including dysbiosis, abnormal gut motility, stress, an altered gut-brain axis, increased mucosal permeability, inflammation or impaired immune function and heightened visceral sensitivity (3). A major factor in the pathogenesis of this condition may be the presence of small intestinal bacterial overgrowth (SIBO). The prevalence of SIBO in patients with IBS symptoms has been reported to be as high as $43-78 \%$ in certain studies (4-6).

In healthy individuals, bacteria colonise the entire length of the gastrointestinal tract, ranging from $10^{1}-10^{3}$ bacteria/g in the stomach to $10^{11}-10^{12}$ bacteria/g in the colon (7). Bacteria in the small intestine are usually Gram-positive aerobes, where the colon is normally colonised by anaerobes. In some cases, conditions within the gastrointestinal tract prohibit natural defence mechanisms to prevent bacteria from overgrowing within the small intestine. This may include, but is not limited to, elevated $\mathrm{pH}$ levels within the stomach or decreased gastric secretions, dysrhythmic activity altering the intestinal motility and an impaired cellular or humoral immune defence or structural issues with the ileocaecal valve (8). These conditions may arise following medications which alter these conditions, such as proton pump inhibitors, antibiotics and anticholinergics, amongst others (7). SIBO involves bacterial overgrowth within the small intestine, usually defined as the presence of $>10^{5}$ colony forming units (CFU)/ml $(9,10)$.

When SIBO is present, carbon dioxide, hydrogen, methane and short-chain fatty acids are produced as a result of bacterial metabolism. These by-products can cause unpleasant abdominal and gastrointestinal symptoms, such as bloating, excessive belching, flatulence, epigastric and abdominal pain, nausea, early satiety, fatigue and altered bowel habits. SIBO has also been associated with increased inflammation and intestinal permeability, where it is linked to the pathogenesis of other conditions, such as non-alcoholic fatty liver disease $(11,12)$. SIBO may lead to an impaired nutrient status by reducing the absorptive capacity of the intestinal villi and the deconjugation of bile salts, leading to decreased fat and fat-soluble nutrient absorption, and competing with the host for vitamin B12 absorption (13). 
The diagnostic test for SIBO is a breath test for the detection of hydrogen and methane gas. These gases are not produced by humans, but are produced by microbial fermentation. These gases diffuse through the gut wall into the circulatory system and are excreted in the breath and therefore can be used as a direct measurement of SIBO, bacterial fermentation and carbohydrate malabsorption. This is a simple and non-invasive procedure although there are limitations (14). Two major groups have convened to standardise the methods and interpretation of breath testing. A number of testing facilities have followed the 2017 North American Consensus recommendations (15) which have been updated in 2019 by the Association of Gastrointestinal Physiology (AGIP) committee of the British Society of Gastroenterology for the UK (16).

The present study describes a case of IBS with a diagnosis of SIBO using a lactulose hydrogen and methane breath test in accordance with the AGIP protocols and guidelines (16). In this patient, SIBO was eradicated using a diet low in fermentable oligo-, di-, mono-saccharides and polyols (FODMAP) and a herbal formulation, which resolved the symptoms of IBS and improved the quality of life of the patient, even following the re-introduction of high FODMAP foods.

\section{Case report}

The patient discussed herein was female at the age of 48 with IBS symptoms described by the patient as severe for at least 10 years. Symptoms included bloating, cramps, flatulence and constipation along with fatigue. Intolerance to garlic was severe, causing vomiting and thus the patient completely avoided its consumption. She also identified other foods as potential triggers, such as cabbage, wheat, lactose and foods high in sugar. The patient had a history of frequent antibiotic use and stress.

Upon her first visit to the Glenville Nutrition Ireland clinic, the patient received a patient code which was used to anonymise all identifying personal data and test results. The patient's personal data and records are securely stored on a clinic system (Clinic Office ${ }^{\mathrm{TM}}$ ) in accordance with the General Data Protection Regulation 2018 (17). In accordance with the regulations, the clinic privacy policy is available online at www.glenvillenutrition.ie. When the case was considered for publication, the patient signed a consent form agreeing for anonymous data, including the test results to be used.

Prior to the initial consultation and at the 6-week follow-up, the patient completed a quality of life questionnaire validated for IBS and functional digestive disorders, which yields a score ranging from 0 (poor quality of life) to 100 (good quality of life) (18). In the initial assessment, the patient's handicap of daily living was deemed to be extreme in the categories of activity, diet and discomfort. Her global score was 42 (Fig. 1).

A strict 24-h preparatory diet of only boiled chicken or poached fish, white rice, eggs and water was followed, including $12 \mathrm{~h}$ of fasting (16). The patient had not taken any antibiotics, laxatives, antacids, proton pump inhibitors or other medications or supplements relating to digestion, such as enzymes or probiotics for at least 1 month. She had begun following a low FODMAP diet at 1 week prior to her initial test due to her own research. At the time of testing, constipation was reduced due to the avoidance of 'trigger' foods and delayed bowel transit time was not apparent.
Table I. Ingredients of the herbal formulation, Nutri Advanced Candex SIBO.

\begin{tabular}{lc}
\hline $\begin{array}{l}\text { Constituents of Nutri } \\
\text { Advanced Candex SIBO }\end{array}$ & $\begin{array}{c}\text { Amount per } \\
\text { capsule (mg) }\end{array}$ \\
\hline Caprylic acid & 167 \\
Grapefruit seed extract & 150 \\
Berberine & 75 \\
Garlic extract & 70 \\
Oregano extract & 50 \\
\hline
\end{tabular}

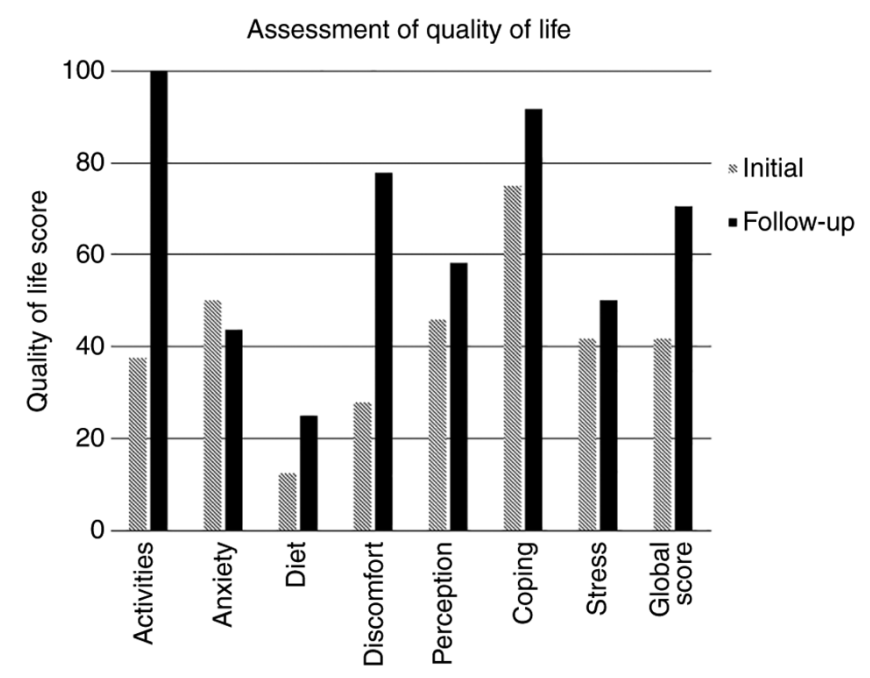

Figure 1. Quality of life score of the patient at the initial appointment and at follow-up following the re-testing of lactulose methane and the hydrogen breath test. The score for each scale ranges from 0 (poor quality of life) to 100 (good quality of life).

SIBO breath testing was carried out at the GastroLife Clinic in Dublin, Ireland using a lactulose substrate in accordance with the AGIP guidelines (16) and was performed using the Bedfont Scientific GastroCHECK ${ }^{\mathrm{TM}}$ gastrolyzer. A positive SIBO test was indicated by a rise in hydrogen gas of $>20$ ppm over the lowest preceding value within $100 \mathrm{~min}$ and methanogenesis was indicated by a rise in methane gas of $>12$ ppm (Fig. 2).

A strict low FODMAP diet was recommended under the supervision of a nutritional therapist using the Monash University FODMAP University App for the most up-to-date food list (19). A herbal supplement termed Candex SIBO (Nutri Advanced; Table I) was used to support the eradication of small intestinal bacteria; the dose of this was slowly increased to two capsules three times per day for 28 days. A mild digestive enzyme was also used before meals to support digestion and motility (Nutri Advanced Marshmallow and Gamma Oryzanol). This contains $120 \mathrm{mg}$ marshmallow root, $65 \mathrm{mg}$ gamma oryzanol and $165 \mathrm{mg}$ mixed digestive enzyme blend per capsule, including amylase, lipase, cellulase, lactase, invertase, peptidase and maltase. It should be noted that the herbal supplements were provided to the patient free of charge by Nutri Advanced. 


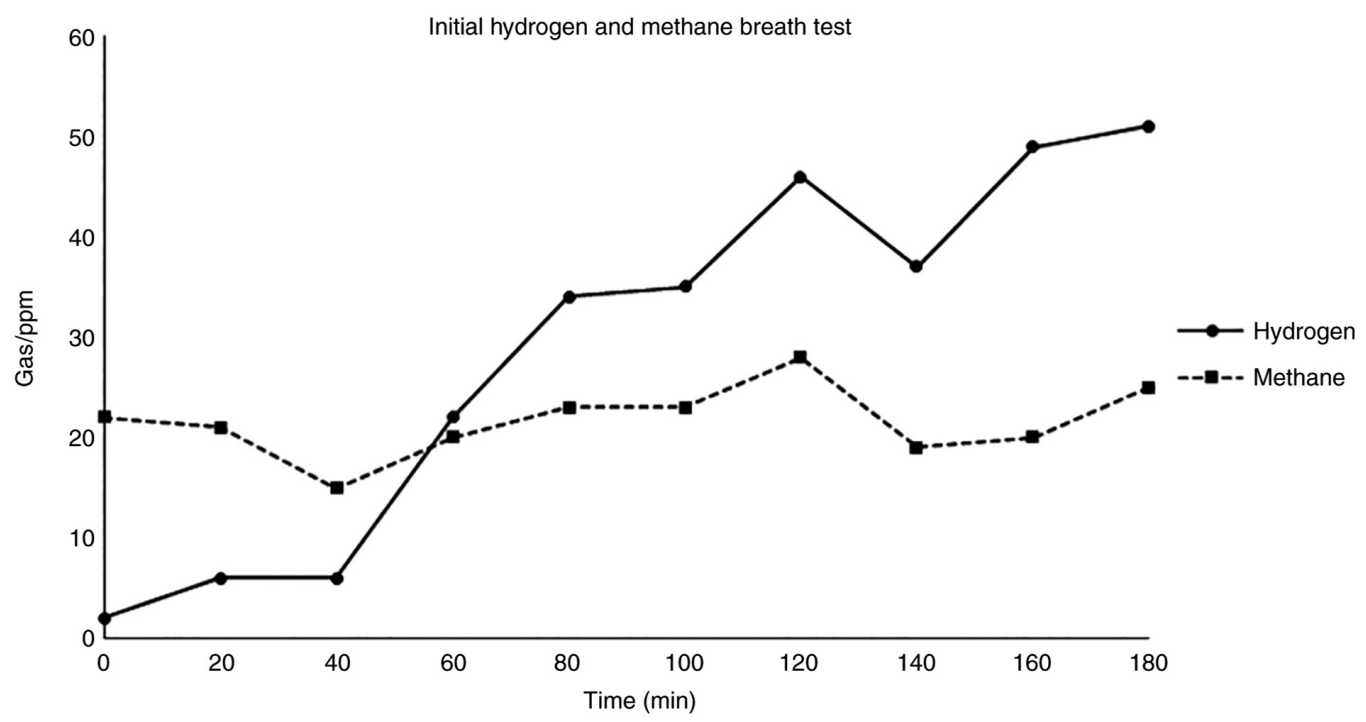

Figure 2. Initial hydrogen and methane lactulose breath test. Hydrogen gas levels were elevated ( $>20 \mathrm{ppm})$ from the 60 -min time point, indicative of small intestinal bacterial overgrowth. Methane gas levels were elevated throughout, confirming methanogenesis.

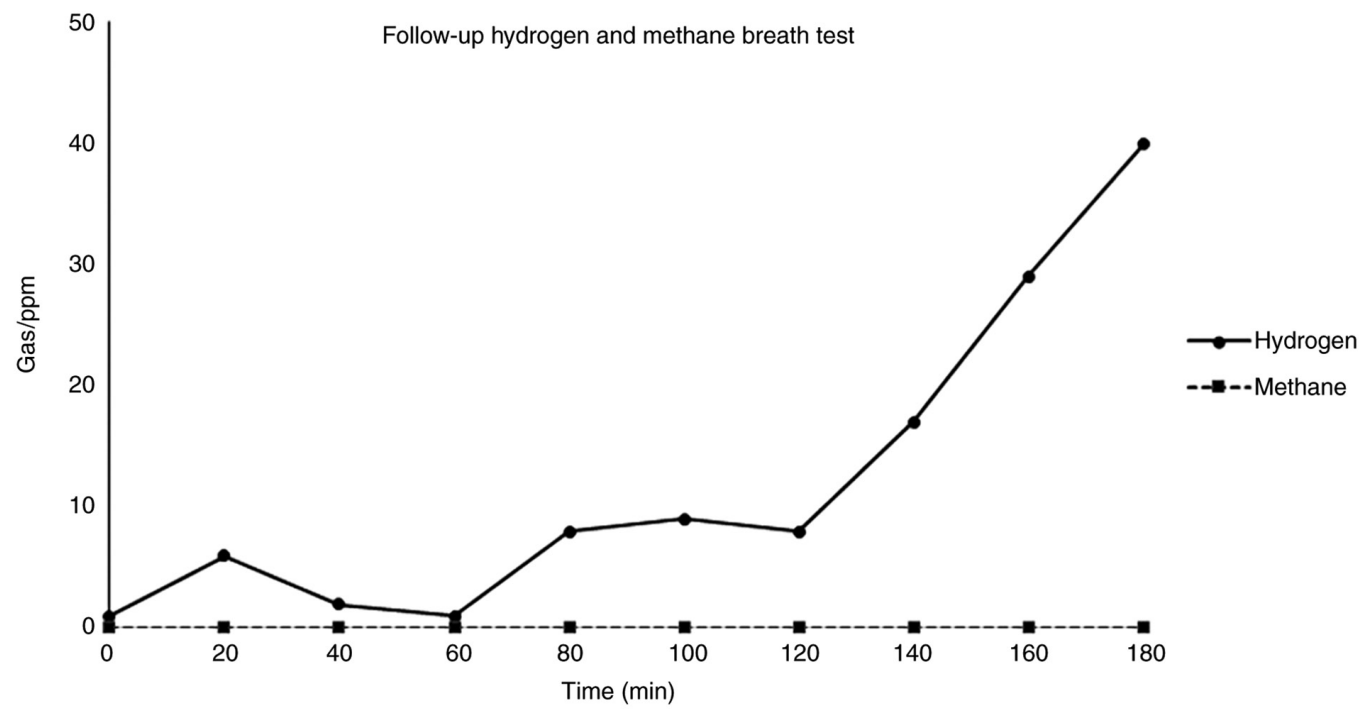

Figure 3. Hydrogen and methane lactulose breath test results at follow-up. Hydrogen gas levels did not increase $>20$ ppm within 120 min, thus indicating that there was no small intestinal bacterial overgrowth. The increase in hydrogen gas levels at $140 \mathrm{~min}$ was expected due to colonic bacteria. There was no methane present in any breath sample over time.

The patient was extremely compliant with the dietary restrictions and supplements. Upon the completion of the 28-day protocol, the patient repeated the breath test within 3 days. The follow-up breath test revealed a marked improvement (Fig. 3). The result was classified as negative for SIBO. Hydrogen gas output indicated normal colonic bacteria and methane was completely absent from the sample throughout. The patient reported feeling considerably better and her quality of life global score increased from 42 to 71 (Fig. 1). Specifically, in the categories of activity and discomfort where she was previously categorised as extreme, there was a marked improvement, where her digestive symptoms no longer had an impact on her quality of life. The score for diet improved to a lesser degree and the score for anxiety did not improve, as the patient was still apprehensive about introducing foods to her diet at the time of testing. The patient had re-introduced wheat, cabbage and had enjoyed a glass of wine. She was counselled to support re-introducing further high FODMAP foods slowly and continued to feel well at the 3-month verbal follow up.

\section{Discussion}

Rifaximin is the most commonly used antibiotic in the treatment of IBS and for the eradication of SIBO. Rifaximin is poorly absorbed and thus has a low systemic activity; however, it is effective against Gram-negative and -positive aerobic and anaerobic bacteria in the digestive tract (20). Clinical studies on SIBO have reported an improvement in IBS symptoms and the normalisation of breath tests in 33-86\% of participants, although these studies differ in diagnostic criteria and methods (21-24). A number of herbal and food-based nutraceuticals have also been used for a number of years for their antimicrobial effects, 
such as garlic, rosemary and cloves (25). There is an increasing awareness of these herbs and nutraceuticals amongst the public, and thus there is an increased demand for complementary approaches to healthcare. In a previous study comparing a combined herbal therapy and rifaximin in patients with a positive lactulose breath test, the response rate to herbal therapy was $46 \%$ compared with one of $34 \%$ to rifaximin $(n=104)(26)$. The herbal formula used in the present case report study, outlined in Table I, was similar to the herbal therapy used in that study (26).

The low FOMDAP diet is the most widely researched diet in the management of IBS. Developed by Monash University, it is low in specific carbohydrates that are categorised as fermentable oligo-, di-, monosaccharides and polyols (19). It can be used to manage the symptoms of IBS; however, its long-term use may be associated with a reduction in dietary quality (27). Only a limited number of studies to date have clearly demonstrated the effect of a low FODMAP diet alone on SIBO. In a previous randomised controlled trial, a 21-day low FODMAP diet did not reduce the area under the curve for hydrogen on the lactulose breath test (28). In another small, yet well-designed study, Ong et al (29) demonstrated that participants (both healthy subjects and patients with IBS) while on a low FODMAP diet produced less hydrogen than those on a high FODMAP diet. There was no lactulose administration during breath testing, which would be a FODMAP challenge. In this crossover design study, participants who had previously reduced their hydrogen output on a low FODMAP diet, had an increased hydrogen output again once they changed to eating a high FODMAP diet. Their digestive symptoms also returned when they ate a high FODMAP diet (29).

Another crossover study analysing colonic bacteria demonstrated that the low FODMAP diet reduced the faecal bacterial growth of Actinobacteria, Bifidobacterium and Faecalibacterium prausnitzii (30). Bacterial growth increased again after a high-fructo-oligosaccharides diet was re-introduced after 3 weeks compared with a placebo. There are thus concerns over the effects of a low FODMAP diet on colonic bacteria and this is one of the reasons why a low FODMAP diet is not a suitable long-term solution for IBS. It is not yet well understood whether a low FOMDAP diet actually reduces small intestinal bacterial overgrowth, although the current available evidence in humans would suggest that it may not, or at least that the effect may be transient.

In the present case report, the low FODMAP diet may have reduced the symptoms of SIBO in the patient; it was suggested that the herbal supplement led to the eradication of the bacterial overgrowth. The improvement in symptoms persisted at 3 months, following the re-introduction of high FODMAP foods, which was confirmed verbally at the follow-up consultation. There is no evidence to suggest that this is a long-term solution and it is widely understood that IBS is a complex condition which may relapse or re-occur. SIBO may also not be the only component of the digestive issues in the patient described herein, although the improvement in symptoms coincided with the normalisation of the lactulose breath test. Further studies with larger patient cohorts are required to investigate the role of SIBO in IBS, to compare the treatment options, such as commonly available herbal options vs. antibiotics with and without a low FODMAP diet, and to determine the long-term outcomes of SIBO treatment.
SIBO is currently under-investigated in patients with IBS despite possibly being a contributing factor to the pathology and aetiology. It is important to bear in mind that testing has limitations; it is recommended that testing be performed in laboratories that strictly adhere to the best available guidelines. It also worth noting that SIBO is not exclusive to IBS, and it is not a factor in all cases of IBS. The present case report may provide valuable insight into SIBO, as it demonstrates that the eradication of this condition using a herbal therapy and a low FODMAP diet reduced the symptoms of IBS and improved the quality of life of the patient, concurrent with the normalisation of the lactulose breath test. However, further studies are warranted to develop an effective treatment protocol.

\section{Acknowledgements}

Not applicable.

\section{Funding}

No funding was received.

\section{Availability of data and materials}

The datasets used and/or analysed during the current study are available from the corresponding author on reasonable request.

\section{Authors' contributions}

CPW assessed the patient, recommended the breath test and designed the protocol. CPW collected the data and drafted the manuscript. MTD performed the breath testing and contributed to the design of the manuscript. HL contributed to the study design and the final revision of the manuscript. All authors confirm the authenticity of all the raw data. All authors have read and approved the final manuscript.

\section{Ethics approval and consent to participate}

The patient signed a consent form for her anonymous data to be used for the purposes of the present study.

\section{Patient consent for publication}

The patient signed a consent form for her anonymous data to be used in this publication.

\section{Competing interests}

MTD is the Director and owner of the GastroLife clinic. CPW and HL are the directors and owners of Glenville Nutrition Clinic Ireland.

\section{References}

1. Canavan C, West $\mathrm{J}$ and Card T: The epidemiology of irritable bowel syndrome. Clin Epidemiol 6: 71-80, 2014.

2. Drossman DA and Hasler WL: Rome IV-Functional GI disorders: Disorders of gut-brain interaction. Gastroenterology 150: 1257-1261, 2016. 
3. Camilleri M: Peripheral mechanisms in irritable bowel syndrome. N Engl J Med 367: 1626-1635, 2012.

4. Pimentel M, Chow EJ and Lin HC: Eradication of small intestinal bacterial overgrowth reduces symptoms of irritable bowel syndrome. Am J Gastroenterol 95: 3503-3506, 2000.

5. Pyleris E, Giamarellos-Bourboulis EJ, Tzivras D, Koussoulas V, Barbatzas $\mathrm{C}$ and Pimentel M: The prevalence of overgrowth by aerobic bacteria in the small intestine by small bowel culture: Relationship with irritable bowel syndrome. Dig Dis Sci 57: 1321-1329, 2012.

6. Carrara M, Desideri S, Azzurro M, Bulighin GM, Di Piramo D, Lomonaco L and Adamo S: Small intestine bacterial overgrowth in patients with irritable bowel syndrome. Eur Rev Med Pharmacol Sci 12: 197-202, 2008.

7. Ghoshal UC, Shukla R and Ghoshal U: Small intestinal bacterial overgrowth and irritable bowel syndrome: A bridge between functional organic dichotomy. Gut Liver 11: 196-208, 2017.

8. Pimentel M, Saad RJ, Long MD and Rao SSC: ACG clinical guideline: Small intestinal bacterial overgrowth. Am J Gastroenterol 115: 165-178, 2020.

9. Corazza GR, Menozzi MG, Strocchi A, Rasciti L, Vaira D, Lecchini R, Avanzini P, Chezzi C and Gasbarrini G: The diagnosis of small bowel bacterial overgrowth. Reliability of jejunal culture and inadequacy of breath hydrogen testing. Gastroenterology 98: 302-309, 1990

10. American Gastroenterological Association medical position statement: Guidelines for the evaluation and management of chronic diarrhea. Gastroenterology 116: 1461-1463, 1999.

11. Miele L, Valenza V, La Torre G, Montalto M, Cammarota G, Ricci R, Masciana R, Forgione A, Gabrieli ML, Perotti G, et al: Increased intestinal permeability and tight junction alterations in nonalcoholic fatty liver disease. Hepatology 49: 1877-1887, 2009.

12. Kapil S, Duseja A, Sharma BK, Singla B, Chakraborti A, Das A, Ray P, Dhiman RK and Chawla Y: Small intestinal bacterial overgrowth and toll-like receptor signaling in patients with non-alcoholic fatty liver disease. J Gastroenterol Hepatol 31: 213-221, 2016.

13. Dukowicz AC, Lacy BE and Levine GM: Small intestinal bacterial overgrowth: A comprehensive review. Gastroenterol Hepatol (NY) 3: 112-122, 2007.

14. Saad RJ and Chey WD: Breath testing for small intestinal bacterial overgrowth: Maximizing test accuracy. Clin Gastroenterol Hepatol 12: 1964-1972, 2014.

15. Rezaie A, Buresi M, Lembo A, Lin H, McCallum R, Rao S, Schmulson M, Valdovinos M, Zakko S and Pimentel M: Hydrogen and methane-based breath testing in gastrointestinal disorders: The north American consensus. Am J Gastroenterol 112: 775-784, 2017.

16. The Association of Gastrointestinal Physiology (AGIP) Committee of the British Society of Gastroenterology (2019). Association of Gastrointestinal Physiologists (AGIP) Proposed Standardised Testing Protocol for Hydrogen/Methane Breath Testing (HMBT) to Assess Small Intestinal Bacterial Overgrowth (SIBO) and Carbohydrate Malabsorption. 2019 British Society of Gastroenterology, (24 June 2019).

17. The European Parliament and the Council of the European Union Regulation: Regulation(EU) 20-16/679 of the European Parliament and of the Council of 27 April 2016 on the protection of natural persons with regard to the processing of personal data and on the free movement of such data, and repealing Directive 95/46/EC (General Data Protection Regulation). Official Journal of the European Union 2018. Available from: https://eur-lex.europa. eu/eli/reg/2016/679/oj. Accessed at November, 2021.
18. Chassany O, Marquis P, Scherrer B, Read NW, Finger T, Bergmann JF, Fraitag B, Geneve J and Caulin C: Validation of a specific quality of life questionnaire for functional digestive disorders. Gut 44: 527-533, 1999.

19. Monash University: The Low FODMAP Diet. Online May 2 , 2019. https://www.monashfodmap.com/. Accessed November 10, 2019.

20. Shayto RH, Abou Mrad R and Sharara AI: Use of rifaximin in gastrointestinal and liver diseases. World J Gastroenterol 22: 6638-6651, 2016

21. Pimentel M: Review article: Potential mechanisms of action of rifaximin in the management of irritable bowel syndrome with diarrhoea. Aliment Pharmacol Ther 43 (Suppl 1): S37-S49, 2016.

22. Meyrat P, Safroneeva E and Schoepfer AM: Rifaximin treatment for the irritable bowel syndrome with a positive lactulose hydrogen breath test improves symptoms for at least 3 months. Aliment Pharmacol Ther 36: 1084-1093, 2012.

23. Sharara AI, Aoun E, Abdul-Baki H, Mounzer R, Sidani S and Elhajj I: A randomized double-blind placebo-controlled trial of rifaximin in patients with abdominal bloating and flatulence. Am J Gastroenterol 101: 326-333, 2006.

24. Pimentel M, Park S, Mirocha J, Kane SV and Kong Y: The effect of a nonabsorbed oral antibiotic (rifaximin) on the symptoms of the irritable bowel syndrome: A randomized trial. Ann Intern Med 145: 557-563, 2006.

25. Lai PK and Roy J: Antimicrobial and chemopreventive properties of herbs and spices. Curr Med Chem 11: 1451-1460, 2004.

26. Chedid V, Dhalla S, Clarke JO, Roland BC, Dunbar KB, Koh J, Justino E, Tomakin E and Mullin GE: Herbal therapy is equivalent to rifaximin for the treatment of small intestinal bacterial overgrowth. Glob Adv Health Med 3: 16-24, 2014.

27. Staudacher HM, Ralph FSE, Irving PM, Whelan K and Lomer MCE: Nutrient intake, diet quality, and diet diversity in irritable bowel syndrome and the impact of the low FODMAP Diet. J Acad Nutr Diet 120: 535-547, 2020.

28. McIntosh K, Reed DE, Schneider T, Dang F, Keshteli AH, De Palma G, Madsen K, Bercik P and Vanner S: FODMAPs alter symptoms and the metabolome of patients with IBS: A randomised controlled trial. Gut 66: 1241-1251, 2017.

29. Ong DK, Mitchell SB, Barrett JS, Shepherd SJ, Irving PM, Biesiekierski JR, Smith S, Gibson PR and Muir JG: Manipulation of dietary short chain carbohydrates alters the pattern of gas production and genesis of symptoms in irritable bowel syndrome. J Gastroenterol Hepatol 25: 1366-1373, 2010.

30. Hustoft TN, Hausken T, Ystad SO, Valeur J, Brokstad K, Hatlebakk JG and Lied GA: Effects of varying dietary content of fermentable short-chain carbohydrates on symptoms, fecal microenvironment, and cytokine profiles in patients with irritable bowel syndrome. Neurogastroenterol Motil: Oct 16 , 2016 (Epub ahead of print). doi: 10.1111/nmo.12969.

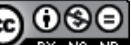

This work is licensed under a Creative Commons Attribution-NonCommercial-NoDerivatives 4.0 International (CC BY-NC-ND 4.0) License. 